\title{
Web-based Application Tool for Recommendation of Open Source Software for Higher Learning Institution in Tanzania
}

\author{
Ambokile Okey \\ The Nelson Mandela African Institution of Science and Technology, \\ P.O. Box 447, Arusha, Tanzania. \\ Email: ambokileokey1990@gmail.com \\ Anael E. Sam \\ The Nelson Mandela African Institution of Science and Technology, \\ P.O. Box 447, Arusha, Tanzania. \\ Email: anael.sam@nm-aist.ac.tz
}

Received: 11 January 2019; Accepted: 29 January 2019; Published: 08 February 2019

\begin{abstract}
Open Source Software (OSS) provide a number of advantages to Higher Learning Institutions (HLIs) in developing countries, such as Tanzania. This is because they are freely available, free to make copies and free to distribute. Through literature reviewed, it has been observed that OSS usage in HLIs in Tanzania is low due to lack of awareness of OSS designed for academic purposes. This paper presents a study on how to implement a reliable and effective web-based application tool for recommendation of OSS for enhancing awareness of HLIs on existing OSS designed for academic purposes. There are three recommendation algorithms that were employed for filtering the OSS in this study: the contentbased, collaborative, and demographic filtering. We used various web technologies for the implementation of application functionalities. The result is the reliable and effective web-based application tool that recommends OSS to individuals in HLIs for enhancing their awareness of OSS designed for academic purposes.
\end{abstract}

Index Terms - Open Source Software, Higher Learning Institutions, Recommendation Algorithms, E-learning.

\section{INTRODUCTION}

The growth of Information and Communication Technologies (ICTs) provide the HLIs with opportunities for researches, learning, and teaching at low cost [1]. It has led to an increase in software tools for facilitating pedagogical activities in HLIs [2]. These software tools can be proprietary, meaning that user must purchase the license to use the software. On the other hand, they can be an open source where users are allowed to make copies, distribute the source codes freely, modify the software so that they fit their organizational or personal needs, and contribute source codes in improving the software. Most of the proprietary software (PS) tools are expensive; this presents challenges to HLIs in Tanzania.
Those challenges include but not limited to budget constraints and illegal use of PS; these hinder HLIs from using software for research, teaching, consultancy, and learning. HLIs can reduce their budget through using OSS; this is very significant for developing countries like Tanzania. Despite the fact that OSS benefit HLIs by easing their pedagogical activities and reduce their budget, many HLIs in Tanzania do not use them due lack of awareness on existing OSS designed for academic purposes [3]. This is because many different choices of OSS are available online, but information about various vendors or platforms that host those OSS is required.

The increase of the number of OSS triggers the need for the web-based application that recommends suitable OSS designed for academic purposes to HLIs. Recommender systems have abundantly been serving in helping users to find useful information when there is information overload online [4].

In Tanzania, many studies provide recommendations to institutions to adopt OSS because of their advantages. Formulation of education policies to support the adoption of OSS is recommended for HLIs in Tanzania [2,3]. OSS products are not always hyped, however, there is vivid evidence of the success of OSS performance which encourages continued use of OSS products in the public and private sectors; this shows the problem of adoption still persist [5]. With the increased use of the Internet, it has become possible to adopt technology for the needs of teaching and learning [6]. Therefore, this study proposes the development of a web-based application tool for the recommendation of OSS for HLIs in Tanzania.

\section{A. OSS in Context}

An OSS is computer software with its source code made available with a license agreement that allows the copyright holder to provide the rights to study, change, and distribute the software [7]. Many countries worldwide insist adoption of OSS in their government sectors because of the free cost of ownership yet 
providing a high level of security, good performance, and reliable service. For instance, Russia makes the use of OSS mandatory in its entire sectors meanwhile others are in the process of adoption [1]. OSS offer several benefits to HLIs as it can be customized to suit the organizational needs. Thus, lecturers and students are no longer forced to use illegal copies of software tools. Eventually, using OSS can contribute to a reduction in running costs, these core benefits are whys for the need and adaption of OSS [7]. OSS in HLIs lies into various categories but not limited to: learning management tools, content management tools, and collaboration tools. Various HLIs Worldwide have adopted OSS for various applications such as in course management and electronic portfolios due to several advantages OSS have over PS [5].

OSS perform a vital role in the success of many HLIs in the world, but many HLIs face a number of challenges. For instance, many people in Oman Sultan Qaboos University are not aware of OSS, leading to its low usage [7].

Some of OSS provide e-learning platforms, the learning management system (LMS) such as Moodle. LMS provides HLIs with a centralized set of tools to help the HLIs succeed in various aspects of curricula such as managing course catalogues, record data from learners, and providing reports to management [8]. In today's education various OSS play a great role in assisting people in HLIs to carry out their tasks, to mention few: Latex for authoring research papers, python for data analysis, android studio for mobile application development, and QGIS for creating, editing, analyzing and publishing geographical information.

\section{B. E-Learning Technologies Adoption Challenges in HLIs in Tanzania}

E-Learning refers to the use of ICTs to enhance and support teaching, learning, and research. This has transformed a traditional way of doing pedagogical activities. E-learning technologies can be in hardware form (Televisions, Digital Versatile Disks (DVDs), Radio) or in software form (E-learning platforms, web-based technologies, software applications) [2]. Software elearning technologies can be proprietary or open source. Albeit the significance of E-learning in education and research its adoption in Tanzanian HLIs though at an increasing rate is apparently low $[5,9]$.

Lack of enough financial resource to budget for elearning tools is a challenge to many HLIs in Tanzania, this is due to the fact that the Government of Tanzania has reduced public funds [10].

OSS tools such as Moodle require Internet infrastructure to operate but other OSS tools such as Latex do not require Internet to operate. There are various challenges faced in utilizing the OSS because as OSS differ in characteristics, so the challenges. Few of OSS tools require the organizational support of ICT infrastructures whilst many do not. However, the ICT infrastructures support is no longer a challenge due to the high rate of growth of Internet connectivity in Tanzania where most of the telecommunication companies offer up to $4 \mathrm{G}$ service to their subscribers [11]. Several OSS tools addressed in this study do not require Internet for them to operate, rather they solely require it during downloading and updating.

Despite the benefits provided by OSS tools, and ICT infrastructure provided by the government of Tanzania, the implementation of OSS tools in HLIs is still low due to a number challenges: lack of systemic approach to ICT implementation, awareness and attitude towards ICTs, administrative support, technical support, staff development, lack of ownership, and inadequate funds [2]. Nevertheless, most of these challenges are not common to all OSS tools, they are most relevant to elearning platforms as they require the involvement of the entire organization.

Generally, the most commonly observed challenge that slows down the adoption process is the knowledge gap between students, academics, system administrators, and lab scientists over the existing e-learning technologies [3].

\section{Recommender Systems Concept}

A recommender system is a software tool and technique providing suggestions for items that may be of use to the user. Users during their online sessions encounter information overload. The recommender systems enrich their decisions on available information. Recommender systems are designed to help individuals who lack sufficient personal experience to evaluate a large number of items that an online platform may provide [4].

There are different classes of recommendation approaches:

1) Content-based: The system learns to recommend items that are similar to ones that user liked in the past. The similarity of items is calculated based on the features associated with compared items.

2) Collaborative filtering: The system recommends to the active user the items that other users with similar taste liked in the past. The similarity in the taste of two users is calculated based on the similarity in the rating history of the users.

3) Demographic: The system recommends items to a user based on their demographic profiles such as age, education, gender etc.

4) Knowledge-based: The system recommends items based on specific domain knowledge about certain item features meet user needs and how that item can be useful for the user. In this system, a similarity function estimates how much the user needs to match the recommendations.

5) Community-based: The system recommends items based on the preferences of users friends.

6) Hybrid recommender system: This system is based on a combination of different techniques mentioned above. This system tries to use the advantages of one technique to fix the disadvantage of another technique. 
This study presents how the web-based application tool for the recommendation of OSS for HLIs is developed using demographic, content-based, and collaborative filtering recommendation techniques. The viability of the developed application would be: to enhance individual awareness in HLIs on existing OSS designed for academic purposes, to improve usage of OSS in HLIs, to improve quality of pedagogical activities through improving usage of OSS designed for academic purposes, and reduce software illegality issues because many people in HLIs in Tanzania are using cracked PS.

The rest of this paper is organized as follows: the second section presents related works. The third section describes the methods used for requirement gathering and development of the web-based application. The design of the web-based application that includes decision-tree of the recommendation process is present in section four. Key functionalities of the web-based application are described in section five. The sixth section presents the results of the implementation of the design and key functionalities. Discussion of results obtained is done in section seven. Finally, the conclusion has been drawn in section eight.

\section{RELATED WORKS}

This section of the paper presents several approaches that have been done to promote and facilitate the adoption of OSS among communities.

\section{A. Alternative Educational Framework}

Alternative education framework which is referred to as a research methodology to identify free and open source software (FOSS); colleges of applied sciences in different regions of the sultanate in Oman used this framework. It is aimed at identifying a list of FOSS to replace PS used in courses of a bachelor degree in information technology (BIT) [1]. This framework presented a list of FOSS for use in BIT, but the list was in a paper-based which may face challenges like storage and ease of access and it was for a single educational area of specialization. In consequence, it needed improvement so that it can be accessed by many people without any restriction, and also includes more academic areas of specialization.

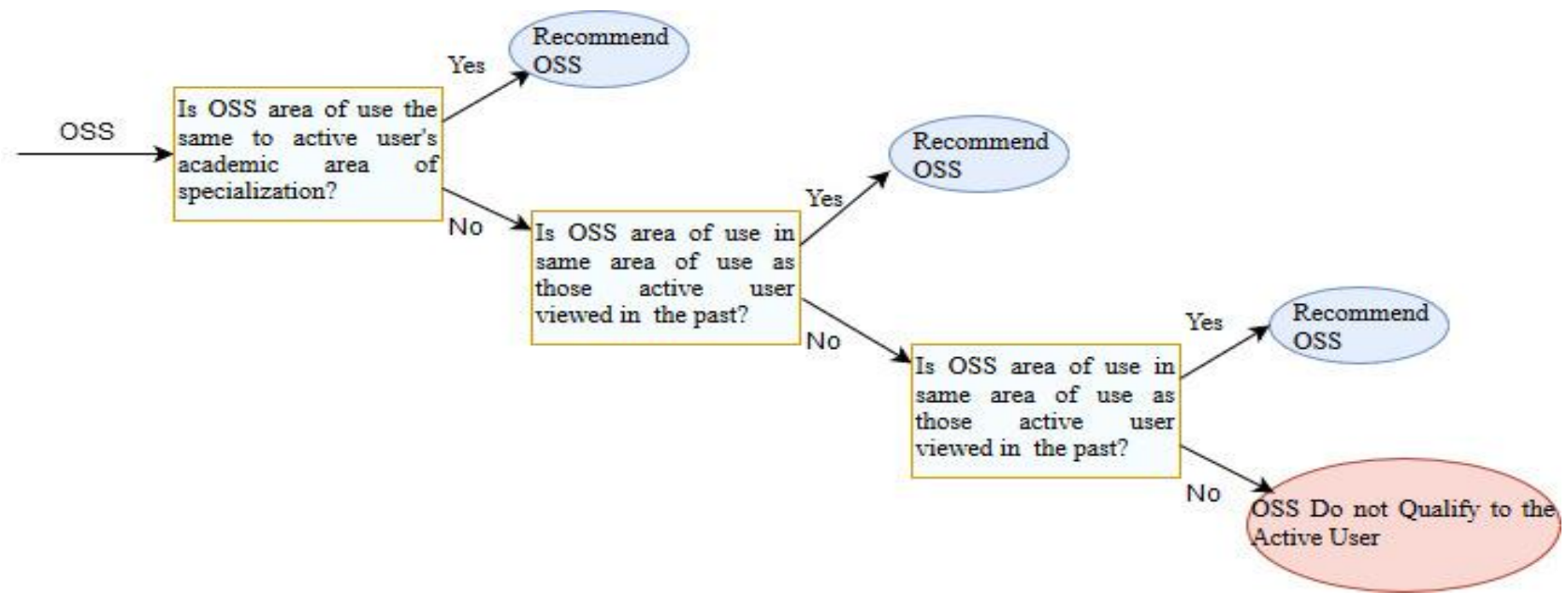

Fig.1. Decision Tree of the Recommendation Algorithms

\section{B. Sourceforge Website}

Sourceforge is a website for publishing open source projects (OSPs). It allows the community to share OSPs by contributing to its improvement. Despite having many projects, Sourceforge treats educational software as a single category, thus there are no recommendations of OSS based on its usefulness to particular pedagogical activity or as an alternative to certain PS to stakeholders in HLIs [12].

\section{Alternativeto Website}

Alternativeto.net is a website that provides alternative recommendations to software. It does not allow direct download rather provide a link to an official website of the software, also like source forge as it treats educational software as a large category as a consequence there are no recommendations of OSS based on its usefulness to particular pedagogical activity or stakeholders in HLIs [13].

\section{METHODOLOGY}

This section of the paper presents the methods used to establish the requirements and development of the webbased application.

\section{A. Requirement Gathering and Analysis}

We used both qualitative and quantitative research methods for requirements gathering. The requirements were analysed and it appeared that majority of the respondents wanted to get recommendations of useful OSS designed for academic purposes, to be able to view and download OSS and to receive email notification about OSS recommendations. However, some requirements were not very clear which led to the 
application of evolutionary prototyping as it allows getting users' feedback during development.

\section{B. Web-based Application Development}

Prototyping is a process of designing and building a scaled-down but working version of the desired system [14]. In this study, evolutionary prototyping was employed, and basic requirements were gathered and analysed. Then the prototype was built and it was improved based on users' feedback. This process was iterative until we got the final version that users were satisfied with.

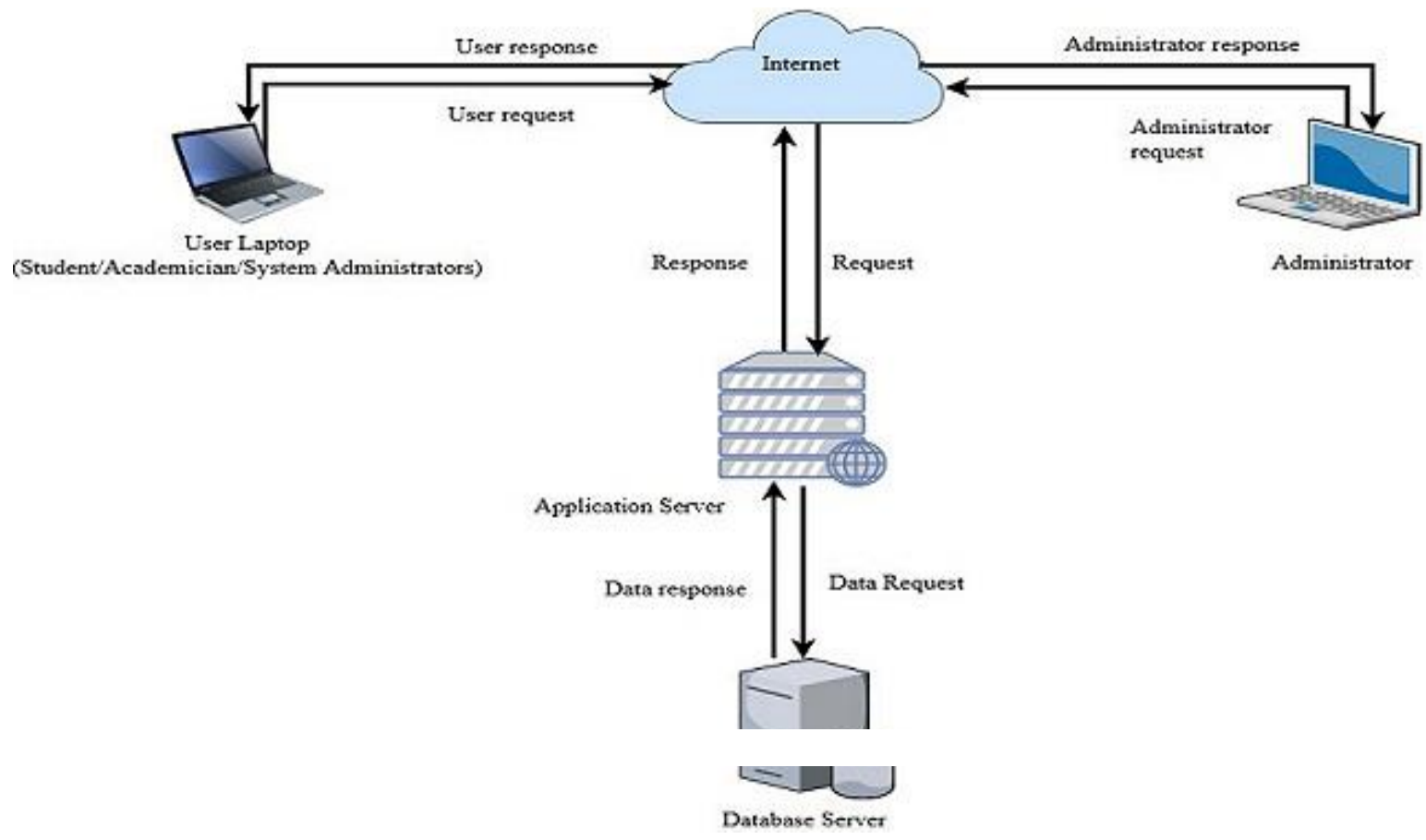

Fig.2. Architecture of the Web-based Application

\section{Design of the Web-BASEd ApPliCATION}

In this section, the design of the web-based application is conducted. It presents a structure of the recommendation algorithms, the architecture of the webbased application and tools used for development.

\section{A. Web-based Application Architecture}

The application runs on a three tiers architecture as shown in fig.2. The application is accessed through the Internet. The users and OSS data are stored on the database server, the application's functions and other application's rules and procedures files are stored in the application server. This ensures more security as the application server checks the request to the database before forwarding to the database server.

\section{B. Structure of the Recommendation Algorithms}

In this subsection, the structure of how the recommendation process works is presented using the decision tree. The application checks if the uploaded OSS academic area of use is the similar to active user's academic area of specialization, or if it is in the same area of use as those the active user viewed in the past, or if it viewed by other users that share academic area of specialization with the active users. The summary of the decisions is shown in fig. 1 .

\section{Tools used for Development}

This subsection of the paper presents web technologies used to implement the application and various server configurations. Web technologies are the collection of technologies that were developed as part of the birth of the world wide web [15]. They are the foundation of the development of web applications.

\section{1) Hypertext Preprocessor}

Hypertext Preprocessor (PHP) is an open-source server-side scripting language, it is used to generate dynamic web pages [16]. PHP can be used together with different web technologies such as Hypertext Markup Language (HTML) and Structured Query Language (SQL). In this study PHP is used to accept data from the client and send them to the database server for storing, to implement different functions that are executed on the server and also to define and enforce security measures.

\section{2) Cascading Style Sheet}

Cascading style sheet (CSS) is used for formatting the web page, it deals with the physical and visual display of the document [17]. CSS can be used as an inline style sheet, inline style attribute or from an external file. The external file gives the best advantage of using CSS as separate document contents and document layout because web developer will need to change only one document to 
control all web pages. CSS is used to format the physical structure of the HTML pages.

\section{3) $H T M L$}

HTML is the main markup language for displaying web pages and other information that can be displayed in the web browser [17]. It is used to generate static contents of the web pages.

\section{4) JavaScript}

JavaScript is the programming language used in HTML pages, it can dynamically modify an HTML page to react with user input or validate user input [18]. JavaScript interaction with the user does not require communication with the server thus it reduces a significant amount of response time the user would send a request to the server.

\section{5) JQuery}

JQuery is a fast, small, and feature-rich JavaScript library, it makes things like HTML document traversal and manipulation, event handling, animation, and Ajax much simpler with an easy to use API that works across a multitude of browsers [19].

6) $M y S Q L$

MySQL is an open source relational database management system whose purposes is to store, manage and retrieve data [16]. MySQL can be used together with other web technologies such as Apache Server and PHP. In this study, MySQL is used as the back-end database, because in this study data are stored as the relationship between entities, each entity was represented as a table. MySQL was selected for implementation because it is open source, it is multi-threaded which enables it to be accessed by multiple users, it can be accessed through the Internet, and it provides support for query language such as SQL which is the standard language for querying the database.

\section{7) Structured Query Language}

Structured query language (SQL) is the standard language for storing, manipulating and retrieving data in databases [16]. SQL is used to communicate with relational databases, in this study, SQL was used to communicate with MySQL for the purpose of storing, managing and retrieving data.

\section{8) Wamp Server}

WampServer is a window web development environment. It allows you to create web applications with Apache2, PHP, and a MySQL database. Apache, MySQL, and PHP can be installed individually and most of the times for the purpose of web development are installed together. WampServer provides a user-friendly way of installing and configure Apache, MySQL and PHP components on Windows. In this study, WampServer 3.0.6 was used as a development environment with Apache version 2.4.23, MySQL version 5.7.14 and PHP version 5.6.25 installed. The following were the procedures for setting up the WampServer

$i$. Downloaded the WampServer through http://www.wampserver.com/en/

ii. The downloaded file was run for installing the WampServer in development computer.

iii. The "www" directory was automatically created in drive C. (c:wamplwww)

$i v$. Then inside "www" folder subfolder was created and application PHP files were kept inside.

$v$. Then on the web browser, this "http://localhost/ResearchSystem/ffoss/pages/" was typed and the server was ready to display web pages.

\section{9) PhpMyAdmin}

phpMyAdmin is a tool written in PHP intended to handle the administration of MySQL over the Web [20]. In this study, PhpMyAdmin was installed together with the WampServer and it was used to manage database, tables, relations, indexes, users, and columns.

\section{0) PHP Configurations}

Default configurations of the Wamp server do not allow large files to be uploaded. Thus PHP was configured so as to allow large files to be uploaded in the server. Those files were the setup files of the OSS. The following were the changes made in the php.ini file found in (C:Iwamp64lbin \php\php5.6.25) to allow files up to 3000MB.

$$
\begin{aligned}
& \text { i. } \quad \text { post_max_size }=3000 M \\
& \text { ii. } \quad \text { upload_max_filesize }=3000 M
\end{aligned}
$$

\section{1) Apache Configuration}

Apache HTTP server is an open source web server developed by the Apache software foundation open source community [21]. In this study, the Apache HTTP server was used to store web contents and accepting user request and send a response to those request by executing PHP scripts. Default Apache configurations do not allow the application to be available over the Internet. Thus Apache was configured so as to make the developed webbased application available over the Internet. The following changes were made in the httpd.conf file and httpd-vhosts.conf which is found in (C:Iwamp64lbinlapachelapache2.4.23 \conf) and (C:Iwamp64lbinlapachelapache2.4.23 \conflextra) respectively.

$i$. The server was configured to listen to any IP address by changing httpd.conf because the server did not have static IP address during development and testing, also the server was configured to listen to port 80 and 8080 .
a. Listen 0.0.0.0:80
b. Listen [: 0]:80
c. Listen [: 0]:8080 
ii. The server was configured to be accessible by any IP address using the following configurations in the httpd-vhosts.conf file. This was done by replacing the line "Require local" with "Require all granted"

\section{Web-BASED APPLICATION FUNCTIONALITIES}

The application functionalities were implemented using the web technologies discussed in the previous section. Users refer to students, academicians, system administrators and lab scientists or any member of HLIs.

\section{A. User Registration}

Users are allowed to use the application with or without registration, the difference is that registered users benefit with more application services. During registration, users are required to provide various information including email and academic area of specialization. The email is verified and used for receiving notification and academic area of specialization was used for recommendation purposes.

\section{B. OSS Uploading}

The application gives privilege to administrator and other users to upload the OSS. They are required to provide important information about the OSS that included a link to the official website, academic area of use, supported operating system (OS), key features and description. The academic area of use is used for recommendation purposes.

\section{User commenting and Rating}

Users are given the ability to comment and rate the OSS after use to help other users of the OSS. The application computes the average rating value (ARV) of each OSS, and it is used to rank the OSS during computations of recommendations.

\section{OSS Recommendations}

OSS recommendations is the main functionality of this application. The academic area of specialization of the user, the academic area of use of the OSS and the ARV is used in the computation in recommendation functions. Three recommendation approaches were used in the application as discussed below.

1) Content-based approach: The application recommends OSS to the active user that are in the same academic area of use as those OSS that user liked in the past. The OSS are then sorted based on the ARV. Fig. 3 presents the algorithm using the flowchart.

2) Demographic approach: The application recommends OSS to the active user when user's academic area of specialization is similar to the OSS academic area of use. The OSS are then sorted based on the ARV. Fig.4 presents the algorithm using the flow chart.
3) Collaborative filtering approach: The application recommends OSS to the active user based on which OSS other users from a similar academic area from specialization have liked in the past. The OSS is then sorted based on the ARV. Fig.5 presents the algorithm using the flowchart.

The three approaches used for recommendations make the application a hybrid recommender application because it uses more than one recommendation approach to recommend OSS to the active users. Further, the application sends a notification to the user when they have a new OSS recommendation.

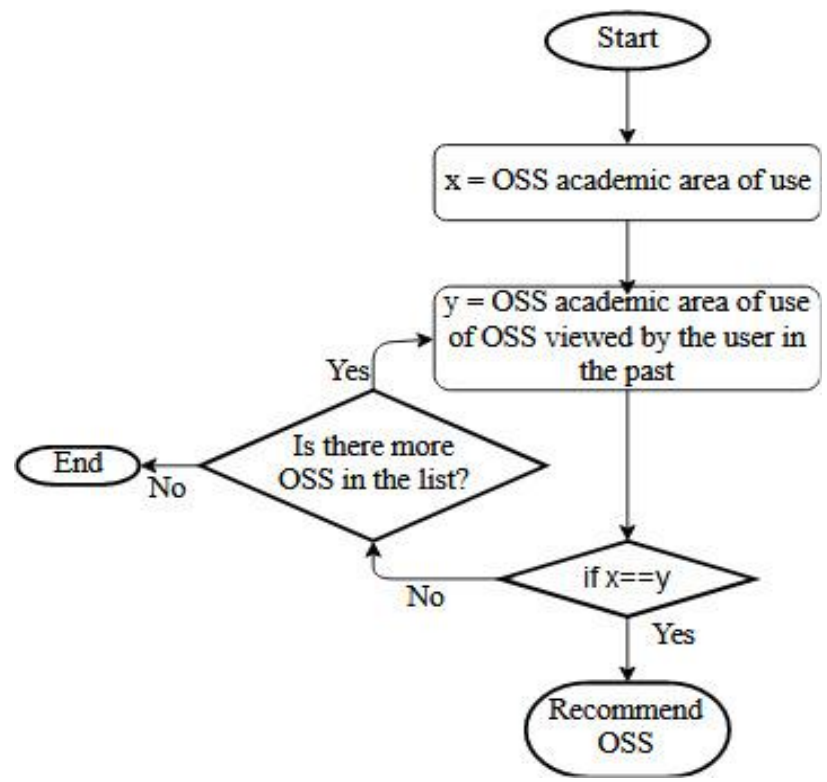

Fig.3. Content-based Approach Algorithm

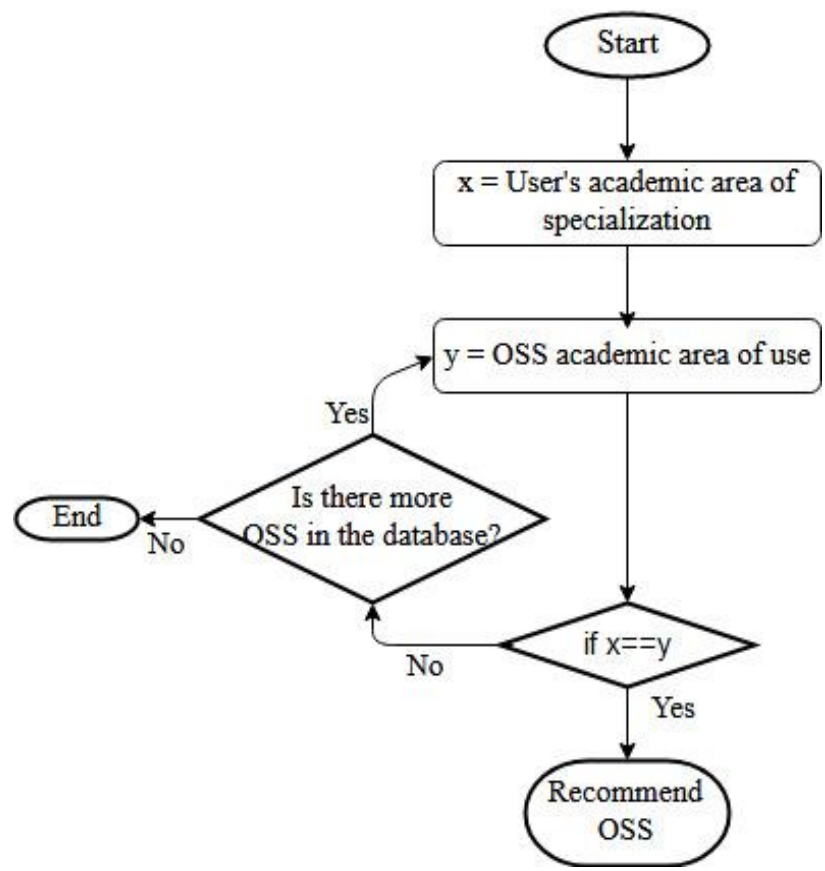

Fig.4. Demographic Approach Algorithm 


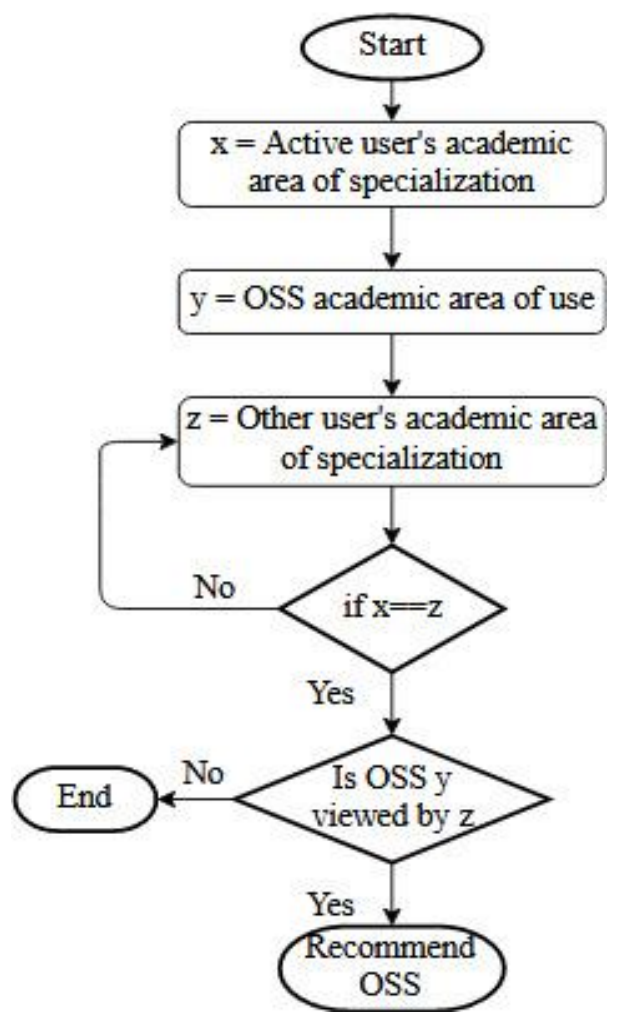

Fig.5. Collaborative Filtering Approach Algorithm

\section{The DeVeloped Web-BASEd APPLICATION}

This section presents some of the functionalities of the developed web-based application. The application provides recommendations of OSS in most of the academic areas of specialization available in Tanzania.

\section{A. Application Homepage}

The homepage of the application is accessible to both registered and unregistered users. The homepage displays the OSS designed for academic purposes and provides links to download and view more details about the OSS such as a description, manual, other users' reviews and ARV of the OSS. Further, the page provides a dashboard that enables users to browse OSS from different academic area of use.

The homepage also provides links to login and registration pages. During registration, a user must provide a valid email address that has to be verified through a link that will be sent to the user' email address provided. The user will set a password that is hashed using an md5 hash, during login user must provide an email address as the username and the password. Fig.6 shows the application homepage.

\section{B. OSS Notification}

The application is implemented to send a notification to users. The user is allowed to select OSS academic area of use to receive notification. Whenever new OSS is uploaded by other users in any of the selected academic area of use, notification will be sent to the user's email address. Fig.7. show one of the notifications sent to the user's email.

\section{OSS Recommendations}

The application provides OSS recommendation to the users as a result of the implementation of recommendation algorithms. The recommendation approaches discussed in section $\mathrm{V}$ of this paper were implemented and the OSS are recommended to the users. The dashboard of the user's page shows a summary of the number of OSS recommended to the user, also the right section of the same page shows a list of the OSS recommended to the user. Fig. 8 shows example on how the application recommended OSS to one of the users.

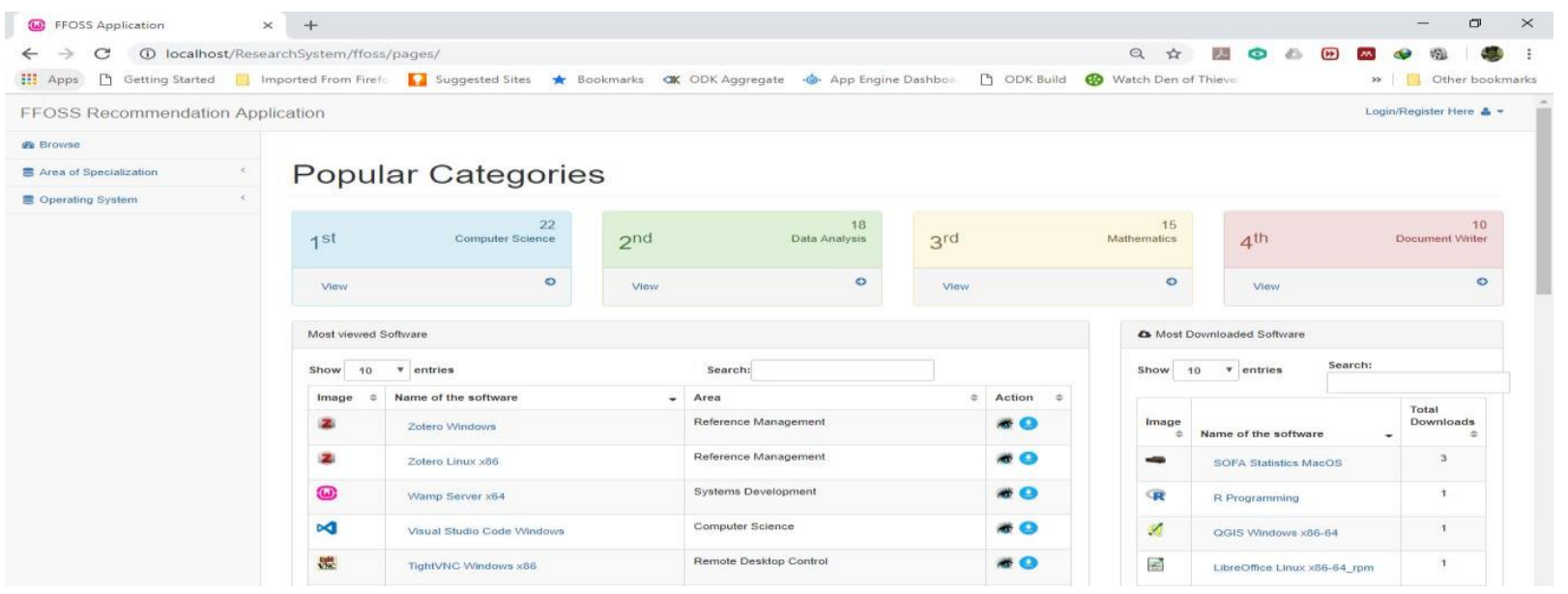

Fig.6. Developed Application Homepage 


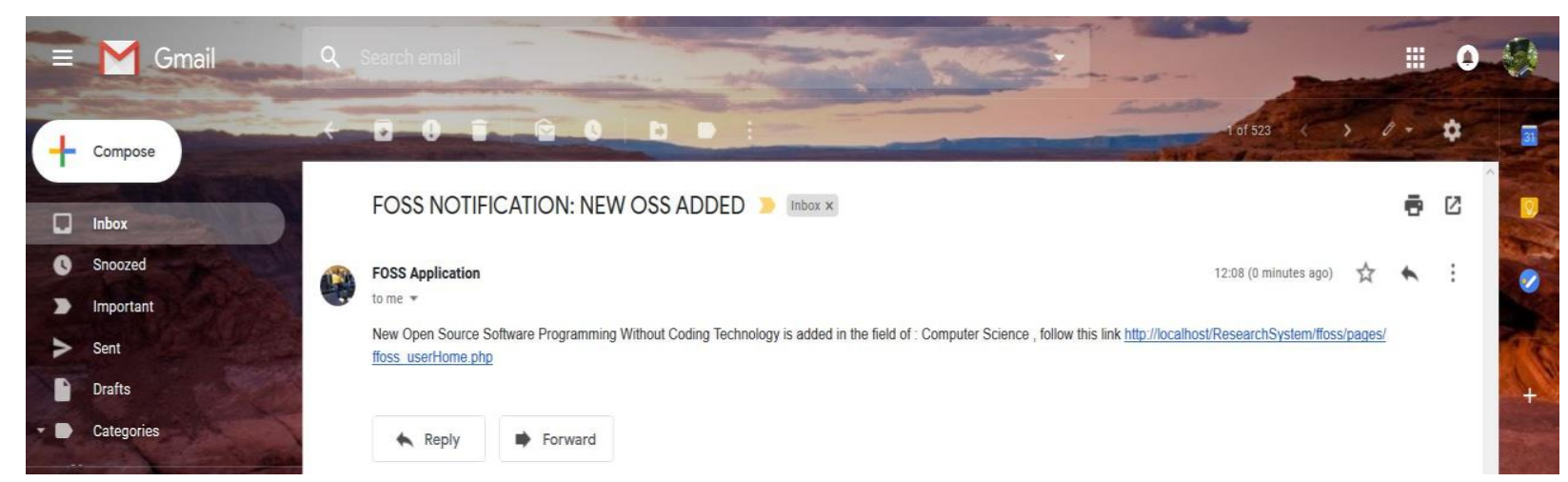

Fig.7. OSS Notification Sent through User's Email

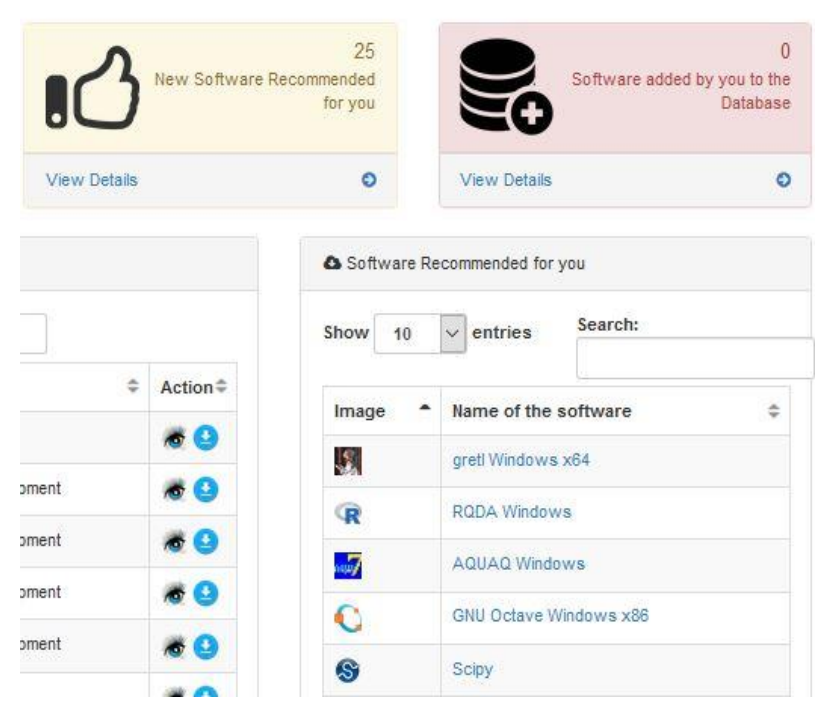

Fig.8. OSS Recommendations

\section{DISCUSSION}

A number of OSS tools to be used for academic purposes were identified, majority of them fall in these areas of use; data analysis, computer science, electronics, telecommunications, software development, network administration, mathematics, document reader and writer, mobile application development, mechanical engineering, image editors, computer drawings, web browser, geographical information systems, accounting, language management and reference management systems. These areas of use are the basis of recommendations provided by the developed application. The number of OSS recommended to the user depends on the recommendation algorithms. Each recommendation algorithm provides advantages but also has disadvantages that are solved by another. For example, the demographic approach cannot provide recommendations of OSS that are viewed by other users or OSS that are in the same area of use as those active user has used in the past, but these disadvantages are achieved by collaborative filtering approach and content-based approaches respectively. Basically, OSS that could not fit in one algorithm criteria may fit in the other. Eventually, this provides a large number of OSS recommended to the active user. This study suggests that hybrid recommender systems are more efficient and reliable in providing recommendations to users than normal recommender systems.

\section{CONCLUSION}

In this study we aimed at improving the adoption of OSS in Tanzanian HLIs through enhancement of individual awareness on the existing OSS designed for academic purposes using the recommendation approaches i.e. content-based, demographic and collaborative filtering. In our study, we nevertheless assessed the level of awareness of various people from HLIs on existing OSS that are designed for academic purposes and the software that are currently used in HLIs. The study, besides these, it explored OSS that can be alternative to currently used software, and more useful OSS that can fit for educational purposes from OSS vendors and other online platforms. Many OSS designed for the academic purpose were found and categorised according to their academic area of use. Eventually, a web-based application tool for the recommendation of OSS was developed by considering the existing situation found during the survey in HLIs. The OSS designed for academic purposes that were found and categorised were uploaded to the application's database and served as a foundation of OSS recommendations. Additionally, the study reviewed and selected proper web technologies that were used for the implementation of application functionalities. The three recommendation algorithms were developed using the web technologies to serve the purpose of recommending OSS designed for academic purposes to the HLIs.

The developed web-based application using the recommendation algorithms is able to; provide recommendations to both registered and unregistered users, send notification about new OSS recommendation through user's email, allow users to share OSS designed for academic purposes with another user through uploading function, allows the user to view and download the OSS and leave their feedbacks.

The ability to recommend OSS designed for academic purposes to its users and send notification through the user's email whenever new OSS is uploaded is a unique feature when compared to similar online platforms. 
The developed web-based application can minimize the search time for OSS online, to minimize HLIs' cost since OSS are freely available and reduce cases of copyright infringements because most of the people in Tanzanian HLIs use cracked PS. We encourage the use of the web-based application for cost-effective selection of software tools for HLIs.

\section{REFERENCES}

[1] R. Al-Hajri, G. Al-Mukhaini, and R. Ramalingam, "Adoption of Free and Open Source Software Using Alternative Educational Framework in College of Applied Sciences," Free open source Softw. Conf., pp. 15-18, 2017.

[2] A. S. Sife, E. T. Lwoga, and C. Sanga, "New technologies for teaching and learning: Challenges for higher learning institutions in developing countries," Int. J. Educ. Dev. Using Inf. Commun. Technol., vol. 3, no. 2, pp. 57-67, 2007.

[3] M. Haule J, "Open Educational Resources Utilization in Tanzania Higher Learning Institutions," Bus. Educ. J., vol. 1, no. 1, pp. 1-28, 2015.

[4] S. Ayushi and V. R. B. Prasad, "Cross-Domain Recommendation Model based on Hybrid Approach," I.J. Mod. Educ. Comput. Sci., vol. 10, no. November, pp. 3642, 2018.

[5] J. H. Lungo and J. J. Kaasbøl, "Experiences of Open Source Software in Institutions : Cases From Tanzania and Norway Experiences of Open Source Software in Institutions: Cases From Tanzania and Norway," in Proceedings of the 9th International Conference on Social Implications of Computers in Developing Countries, 2007, no. May 2007.

[6] B. S. Reddy, "Analysis of Learning Management Systems by Extracting Effectiveness Factor wise," J. Eng. Educ. Transform., 2018.

[7] A. S. Al-Badi and A. H. Al-Badi, "Open Source Software in Higher Education: Oman SQU Case Study," Inf. Technol. Educ. 21st Century, vol. 8, no. 8, pp. 116-124, 2007.

[8] Z. Fariha and A. Zuriyati, "Comparing Moodle and eFront Software for Learning Management System," Aust. J. Basic Appl. Sci., vol. 8, no. 4, pp. 158-162, 2014.

[9] L. John and A. E. Sam, "An Online Collaborative Discussion Platform for Bridging a Technological Reliance Gap in Higher Learning Institutions in Tanzania," Int. J. Mod. Educ. Comput. Sci., no. November, pp. 29-35, 2018.

[10] E. Lwoga, "Making learning and Web 2.0 technologies work for higher learning institutions in Africa," CampusWide Inf. Syst., vol. 29, no. 2, pp. 90-107, Mar. 2012.

[11] TCRA, "Quarterly Communications Statistics," Dar es Salaam, 2018.

[12] Slashdot Media, "SourceForge - Download, Develop and Publish Free Open Source Software." [Online]. Available: https://sourceforge.net/. [Accessed: 12-May-2018].

[13] Ola and markus, "AlternativeTo - Crowdsourced software recommendations." [Online]. Available: https://alternativeto.net/. [Accessed: 12-May-2018].
[14] J. S. Valacich, J. F. George, and J. A. Hoffer, Essentials of Systems Analysis and Design, Fifth. New Jersey: Pearson Education, Inc, 2012.

[15] J. C. Jackson, Web Technologies: a computer science perspective. New Jersey: Pearson Education, Inc., 2007.

[16] C. Darie and E. Balanescu, E-Commerce Beginning PHP and $M y S Q L$, Second Edi. United States of America: Apress, 2008.

[17] R. Nixon, Learning PHP, MySQL, JavaScript, and CSS, 2nd Editio. Sebastopol: O'Reilly Media Inc, 2012.

[18] D. Odell, Pro JavaScript RIA Techniques. New York: Apress, 2009.

[19] “jQuery.” [Online]. Available: https://jquery.com/. [Accessed: 04-Aug-2018].

[20] N. Ibennetch, "phpMyAdmin download SourceForge.net." [Online]. Available: https://sourceforge.net/projects/phpmyadmin/. [Accessed: 15-Aug-2018].

[21] Apache Software Foundation, "Welcome! - The Apache HTTP Server Project," 1995. [Online]. Available: https://httpd.apache.org/. [Accessed: 14-Sep-2018].

\section{Authors' Profiles}

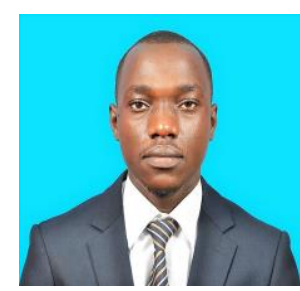

Ambokile Okey is a Tutorial Assistant at the Dar es Salaam University College of Education (DUCE, Dar es Salaam, Tanzania). He obtained his Bachelor Degree of Science with Computer Science in 2014 from the University of Dar es Salaam. Currently, he is pursuing Master's Degree in Information and Communication Science and Engineering (ICSE) at the Nelson Mandela African Institution of Science and Technology (NM-AIST, Arusha, Tanzania)

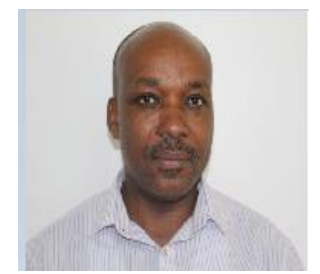

Anael E. Sam is a Senior Lecturer in the Department of Communication Science and Engineering, School of Computational and Communication Sciences and Engineering (CoCSE), at the Nelson Mandela African Institution of Science and Technology. Dr Anael E. Sam is a holder of PhD in Electronics Engineering from the Institute of Electronics and Photonics, Faculty of Electrical Engineering and Information Technology, Slovak University of Technology, Bratislava, Slovak Republic. His research interests are in Mobile and wireless communications, Software Quality Assurance Engineering and Cyber Security.

How to cite this paper: Ambokile Okey, Anael E. Sam, "Web-based Application Tool for Recommendation of Open Source Software for Higher Learning Institution in Tanzania", International Journal of Modern Education and Computer Science(IJMECS), Vol.11, No.2, pp. 33-41, 2019.DOI: 10.5815/ijmecs.2019.02.05 